\title{
Introduction to the Global Health IT Strategies Minitrack
}

\author{
Joseph Tan \\ McMaster University \\ tanjosep@mcmaster.ca
}

\author{
Michael S. Dohan \\ Lakehead University \\ msdohan@lakeheadu.ca
}

\author{
Ofir Turel \\ California State University, \\ Fullerton \\ oturel@exchange.fullerton.edu
}

Global advancements in information and communication technologies have provided opportunities to address issues important to global health, such as coordinating responses to emerging diseases, and addressing health issues that have an international interest throughout the world. These technologies assist in disease surveillance and response, global health education, applying traditional telemedicine applications, as well as other applications for all healthcare actors. These efforts are already impacting the rapid growth and further development of global healthcare solutions and approaches arising from the active collaboration among cross-disciplinary researchers, multi-national agencies and international clinical practitioners.

This minitrack covers broad issues related to Global Health IT strategies, such as applying technology to address health issues that have a global impact. Topics include disease surveillance and response, global health education, community health, coordination of healthcare efforts internationally, new forms and modalities of care delivery aided with Health IT globally such as use of innovative lowcost, mobile and sensor-enabled and other emerging health technological applications. These solutions will provide a multinational perspective on the benefits of mobile health and other emerging information technologies and describes different examples and applications implemented.

For the $50^{\text {th }}$ anniversary of HICSS, our minitrack will cover many interesting and timely topics. First of all, the innovative use of publicly available datasets presents many opportunities to address global health issues. There seems to be much promise in using these widely available datasets for the applications such as the characterization of diseases and monitoring of outbreaks. Two papers in this minitrack take this approach to address two very different global health issues. The first, "Analysis of ZIKA Virus Tweets: Could Hadoop Platform Help in Global Health Management?", finds that a Big Data approach to analyzing social media data was useful for characterizing the Zika disease, and provides direction for future research. Another paper, "Data Visualization on Global Trends on Cancer Incidence", applies visual analytics to a publicly available World Health Organization dataset that consolidates over 100 cancer registries worldwide. They present visualizations of this data where certain global trends can be detected.

Several emerging technologies have had an impact on areas outside of healthcare. Applications of these innovative technologies are starting to take the interest of healthcare researchers and practitioners, and their global health applications are emerging. Crowdsourcing often entails the use of communication technology to source knowledge for a problem, and can be applied to accessing a global network of physicians to diagnose rare conditions. The paper "Developing Effective Crowdsourcing Systems for Medical Diagnosis" outlines challenges and recommendations for the design and features of the technology intended for this purpose. The paper, "Drone Delivery Models for Healthcare", presents an analysis of potential business models for delivering healthcare supplies and devices to underserved areas.

Finally, this minitrack contains research on interventions for healthcare systems improvement that have global applications. "Community Health Workers as Innovators" reports on a pilot program that helps educate community health workers on finding funding for their own innovative projects that will serve urban low-income populations that are underserved. "A Hybrid Mining Approach to Facilitate Health Insurance Decision" offers an approach for single-payer systems to gain efficiencies with claim reviews.

This is the fourth year that the Global Health IT Strategies minitrack has appeared at HICSS, and this year sees an increase in the papers submitted to this minitrack. In all, this minitrack will have seen the presentation of 15 papers, some of which have been accepted for publication in the International Journal of Healthcare Information Systems and Informatics (IJHISI). 